Pure Appl. Chem., Vol. 72, No. 12, pp. 2265-2274, 2000.

(C) 2000 IUPAC

\title{
Adventures in molecular recognition. The ins and outs of templating ${ }^{*}$
}

\author{
Jeremy K. M. Sanders \\ University Chemical Laboratory, Lensfield Road, Cambridge CB2 1EW, UK
}

\begin{abstract}
Two different approaches are described for the creation of supramolecular systems potentially capable of recognition and catalysis. Using the design approach, we have been able to accelerate and influence two different Diels-Alder reactions within the cavities of porphyrin dimers and trimers; this is templating from the outside inwards. The selection approach is a synthetic chemical attempt to capture some of the key evolutionary features of biological systems: dynamic combinatorial chemistry is used to create equilibrating mixtures of potential receptors, and then a template is used to select and amplify the desired system. Five potential reactions for such dynamic chemistry are discussed: base-catalyzed transesterification, hydrazone exchange, disulfide exchange, alkene metathesis, and Pd-catalyzed allyl exchange, and preliminary templating results (inside outwards) are presented.
\end{abstract}

\section{INTRODUCTION}

Molecular recognition and templating are key components of the emerging science of supramolecular chemistry. Supramolecular chemistry is, in Jean-Marie Lehn's words, "Chemistry beyond the molecule", and its goal is to gain control over the intermolecular noncovalent bond. It is concerned with the organization and properties of ensembles of molecules held together by weak interactions: host-guest complexes, self-assembled objects, and spatially organized films can all be conjured out of small building blocks by the careful exploitation of hydrogen bonds, metal-ligand binding, $\pi-\pi$ interactions, and hydrophobic effects. The resulting ensembles have potential as catalysts, sensors, or new materials. The principles and underlying interactions are not new, but the bringing together of such disparate elements to form an emerging, coherent discipline that crosses conventional boundaries is relatively new.

We want to use supramolecular chemistry to construct synthetic receptors that are capable of recognition and catalysis. We have been following two complementary approaches: Design, where one decides on the target host system to be made, makes it, then studies its properties; and Selection, where one generates a large number of related structures by a random or combinatorial process, and then selects the best. Synthetic chemists have so far concentrated on design while selection approaches have historically been dominated by biological systems such as catalytic antibodies or ribozymes. We have recently reviewed the area of supramolecular catalysis, its successes, failures, and future [1]. The common feature of all the work described here is that it involves templating, either from outside in (the first section) or from inside out (the second section).

\section{THE DESIGN APPROACH}

For a variety of reasons, we find metalloporphyrins attractive building block(s) to use [2,3]: variation of the central metal ion changes the recognition specificity, while the extended aromatic $\pi$-system leads

*Plenary lecture presented at the $15^{\text {th }}$ International Conference on Physical Organic Chemistry (ICPOC 15), Göteborg, Sweden, 8-13 July 2000. Other presentations are published in this issue, pp. 2219-2358. 
to characteristic NMR and UV/visible spectroscopic properties. We will use just one type of recognition event here, the labile coordination of pyridine by $\mathrm{Zn}$ porphyrins; the spectroscopic consequences of such a binding event are summarized in Fig. 1.

Using alkyne linkers between the porphyrins and oligopyridine templates to organize the building blocks, a variety of linear and cyclic oligomers have been prepared [4,5], and their binding properties studied [6].

These hosts were designed to act as templates for the Diels-Alder reaction (i.e., as Diels-Alderases as shown in Fig. 2), where the undefined linker is variable. Controlling bimolecular reactions is difficult because two substrates need to be recognized and correctly oriented at the same time. In a fusion process like the Diels-Alder reaction there is the additional problem that turnover is likely to be inhibited by strongly bound product unless a geometry-modifying event can be engineered after the host-catalyzed step. However, one can still accelerate the reaction and influence regio- or stereochemistry, using stoichiometric amounts of host to direct the outcome of the reaction. We discovered that subtle changes in host structure can lead to dramatic changes in the stereochemical outcome of the Diels-Alder reaction, where two finely balanced pathways compete as shown in Fig. 3 [5]. At $30^{\circ}$, in

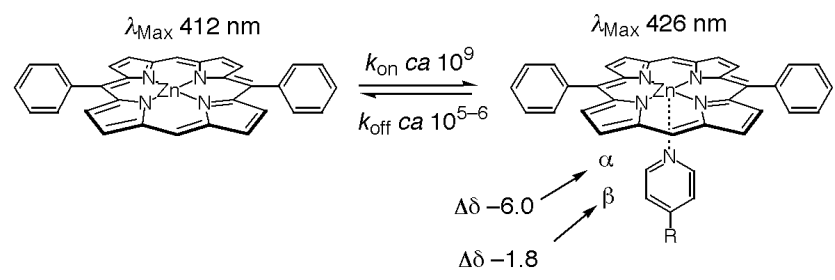

Fig. 1 Complexation of a $\mathrm{Zn}$ porphyrin with pyridine, together with the key spectroscopic consequences; $\Delta \delta$ is an upfield shift in ppm.

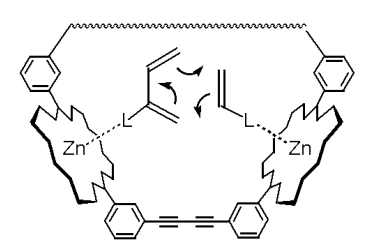

Fig. 2 A series of Diels-Alderases?

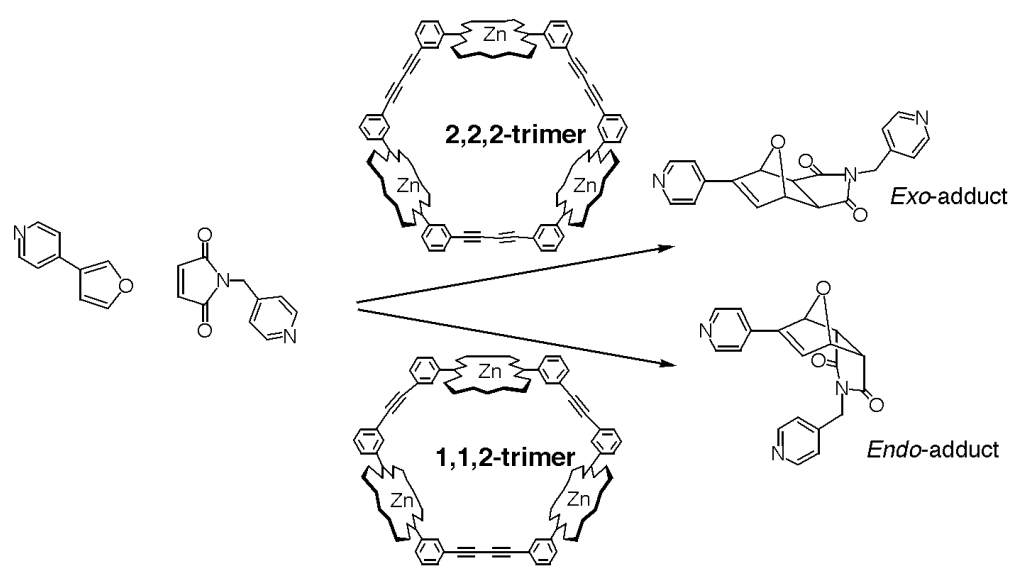

Fig. 3 Redirection of a Diels-Alder reaction using the geometrical constraints of a host cavity [5]. 
the absence of hosts, exo and endo adducts are produced in roughly 2:1 proportion; exo adduct is the thermodynamic product in this reversible Diels-Alder reaction. At $30^{\circ}$ and $0.9 \mathrm{mM}$ concentration in nonpolar solvents and in the presence of one equivalent of 2,2,2-trimer, exo adduct is produced exclusively and with acceleration of more than 1000-fold, while 1,1,2-trimer leads to endo adduct only, and 500 -fold acceleration.

The reversal of stereoselectivity between the two cyclic trimers at $30{ }^{\circ} \mathrm{C}$ is the result of two separate effects, one predicted and one not: the large endo acceleration induced by the smaller 1,1,2-trimer was expected, but the fact that this smaller trimer is ineffective at $30^{\circ} \mathrm{C}$ at accelerating the exo reaction and binding the exo adduct was a surprise. The key difference appears to lie in the greater flexibility of the larger 2,2,2-system: neither of the trimer hosts has the ideal equilibrium geometry to bind the exo transition state or adduct, but at $30^{\circ} \mathrm{C}$ the larger trimer is more flexible and so is better able to respond to the geometrical demands of the exo pathway. One might claim that the stereochemical reversal at $30{ }^{\circ} \mathrm{C}$ is a major success for the design approach, but it is important to note that at $60{ }^{\circ} \mathrm{C}$ the small trimer becomes more flexible and loses its stereoselectivity. As in templated synthesis, which depends crucially on concentration effects, and in many other areas of science, there is a narrow line between spectacular success and dismal failure [4].

The even more difficult tasks of changing regiochemistry and engineering catalytic turnover remain a challenge to be addressed. In an attempt to achieve turnover, we turned to the hetero Diels-Alder reaction shown in Fig. 4. The first step should be accelerated by our porphyrin oligomers, while the second step should lead to a dramatic geometry change, weaker binding, and, therefore, catalytic turnover. We have been unable as yet to achieve the second step (and therefore turnover) cleanly in the presence of porphyrins, but the first step is successfully accelerated by the same trimers as above and by a range of capped dimers $[7,8]$.

We have obtained tiny crystals of these new dimer hosts with and without the Diels-Alder adduct in the cavity and have obtained crystal structures using a synchrotron X-ray source [8,9]. We are trying to build up a complete structure-activity relationship by combining solution-state kinetics and thermodynamics with crystal structures; Fig. 5 shows how just one host is distorted by product formation, despite the fact that it is capable of accelerating the reaction.

Using this design (or, more realistically, trial and error) approach we have learned a great deal about matters such as the role of flexibility in achieving acceleration. For example, although host preorganization is an important contributor, it is also important that the host geometry is responsive to the spatial demands of the transition state. Remarkably, the linear dimer shown in Fig. 6 accelerates both of the above Diels-Alder reactions surprisingly efficiently and is entirely exo-selective in the first: had

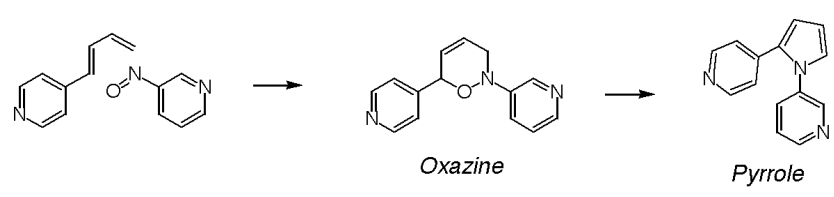

Fig. 4 Hetero Diels-Alder reaction with possible subsequent rearrangement.
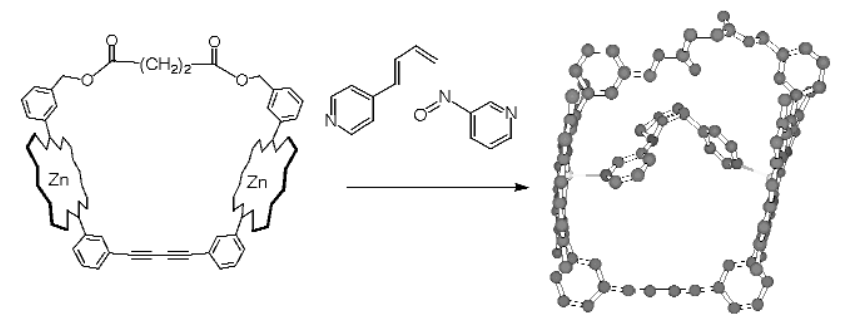

Fig. 5 Product-induced distortion of metalloporphyrin host [8].

(C) 2000 IUPAC, Pure and Applied Chemistry 72, 2265-2274 
we discovered this before making the cyclic trimers we would have been delighted and astonished by how good it is.

However, a major disadvantage of the design approach is that the synthesis of each new oligomer takes a large amount of effort, and, of course, the success rate for rate acceleration by each newly designed system is rather low. In the long term, a more rapid and versatile synthetic approach is required, and this is described now.

\section{THE SELECTION APPROACH}

Given that enzymic catalysis implies selective recognition of the transition state, a suitable transitionstate analog (TSA) should be able to elicit or select good catalysts from a mixture of many different molecules. Current approaches sharing this idea include molecular biology, organic and inorganic synthesis, and polymer and solid-state chemistry; these have been reviewed elsewhere [1,10]. We have been exploring a synthetic chemistry approach using dynamic combinatorial libraries (DCLs). In a traditional combinatorial library, the covalent bonds between building blocks $A, B, C \ldots .$. are fixed during synthesis; there is no post-synthetic interconversion between members such as $A B C$ and $A C B$. In a dynamic combinatorial library, the connections between building blocks are reversible, continuously being made and broken (Fig. 7); these connections may be covalent bonds, or they may be noncovalent metal-ligand or hydrogen-bonding interactions [11]. The composition of a DCL will be dependent on its environment: addition of a template $\mathbf{T}$ that selectively binds one member will bias the equilibrium towards that member, while a different template $\mathbf{T}^{\prime}$ will bias the composition differently.

Our approach is summarized in Fig. 8: a mixture of building blocks will be combined to create the optimum host system for any chosen TSA: the TSA selects from the library those components that

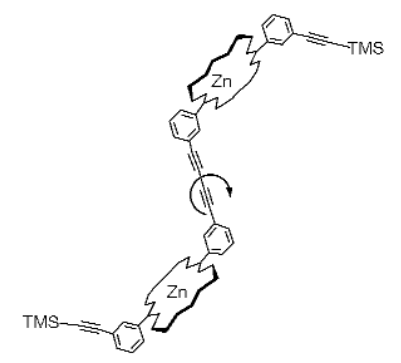

Fig. 6 Rotationally flexible porphyrin dimer.

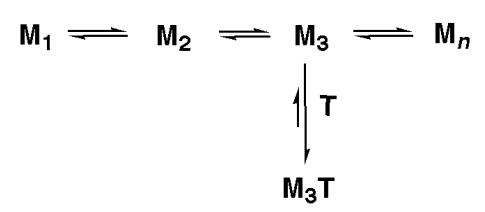

Fig. 7 Templating in a dynamic combinatorial library.
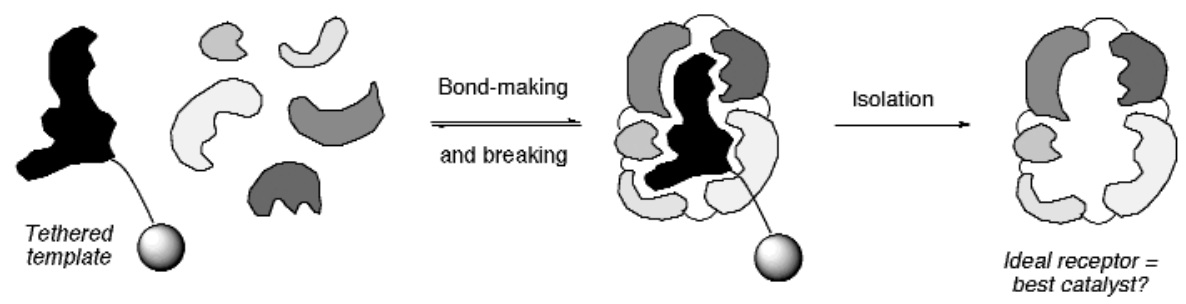

Fig. 8 The general selection scheme. 
bind it best. Under thermodynamic conditions, the equilibrium will be driven toward the formation of hosts for the TSA template, while any incorrect bond-making will be proofread and rejected. The TSanalog will be tethered to a bead and will readily be isolated from the mixture together with its ideal, catalytically active host. The same approach is applicable to removing unwanted molecules from industrial effluent or for sensors.

The building blocks required for this work will be bifunctional, as shown in Fig. 9; we tend to prefer those with a concave shape that encourages macrocycle formation, and we generally use natural products such as steroids, alkaloids, and amino acids as they are cheap, homochiral, and readily functionalized with spectroscopic reporters and recognition functions.

The ideal reversible reaction needs to use functionalities which are readily incorporated into building blocks, to be easy to initiate and to arrest, and rapid in its approach to equilibrium even though the concentration of $\mathrm{X}$ and $\mathrm{Y}$ in Fig. 9 is necessarily equal. For thermodynamic templating to be effective, the intrinsic energy differences between possible products need to be minimal, and the best way to achieve this is to ensure that there is no net change of functionality, that is, through exchange reactions.

Initially, we used base-catalyzed transesterification (Fig. 10a), each cholate building block being equipped with a methyl ester and a hydroxyl group [12]. Reaction in refluxing toluene is initiated by catalytic methoxide and is driven to macrocycle formation by azeotropic removal into molecular sieves of the initially formed methanol. In this way, we could generate mixtures in which each oligomer is a conventional, robust organic molecule which can be isolated and purified, but which on being placed into fresh reaction mixture, reverts to the equilibrium distribution. These are, therefore, dynamic systems, with building blocks continually and promiscuously changing partners. We then widened the repertoire of building blocks to cinchona alkaloids [13,14]. Macrocycle formation was efficient, but led to strong preferences for particular geometries: quinine-derived monomers gave almost quantitative for-

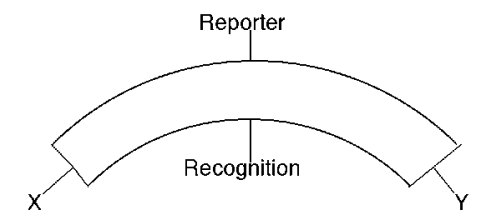

Fig. 9 A general building block.

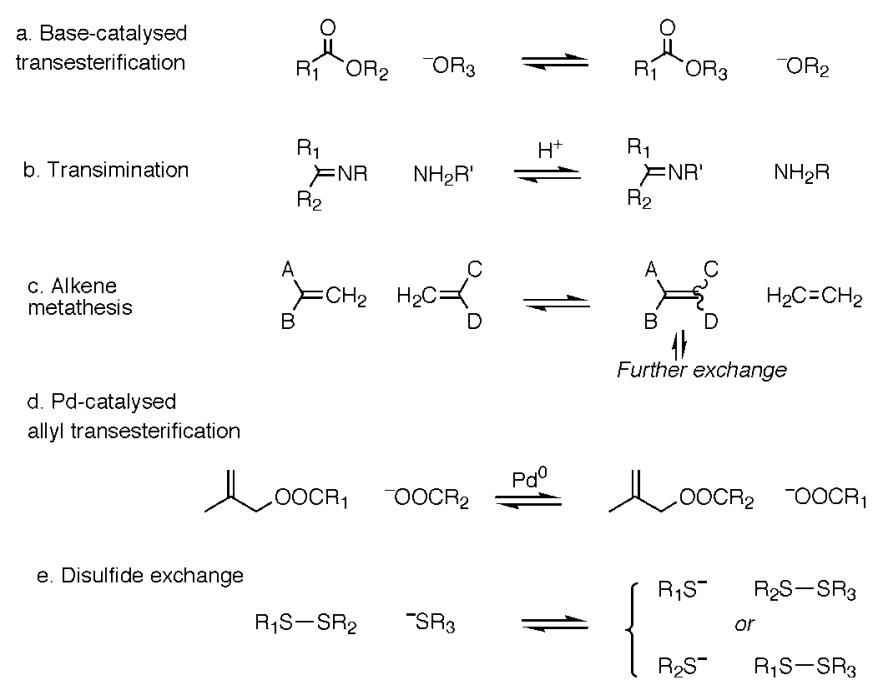

Fig. 10 Some candidate reactions for dynamic combinatorial chemistry.

(C) 2000 IUPAC, Pure and Applied Chemistry 72, 2265-2274 
mation of cyclic trimers, while a quinidine monomer gave cyclic dimer. Unambiguous synthesis using conventional chemistry proved that other ring sizes are kinetically accessible: when a product is thermodynamically favored, the best way of making it is actually through reversible chemistry. Combinatorial diversity (i.e., formation of oligomers of a range of sizes containing mixtures of building blocks) could be engineered by use of flexible chain extensions as in an "extended" quinine [14] or small "facilitator" building blocks derived from ephedrine [13]. Despite very considerable effort, the only convincing templating observed using these transesterification conditions has been with metal ions [12]. The hostile environment, involving high temperatures, aggressive methoxide catalyst, and rigorous exclusion of water, is not conducive to subtle exploitation of noncovalent interactions.

Imine exchange (Fig. 10b) is much more promising and is being explored by other groups as well as ourselves [11]: we are particularly interested in exploring hydrazone exchange as it is turned on by catalytic acid, turned off by base, and gives products which are capable of peptide-like hydrogen bonding $[15,16]$. Figure 11 shows our current general scheme and building block design. The hydrazone formation and exchange chemistry takes place under mildly acidic conditions at room temperature over a period of minutes to hours in a range of solvents and without the need for rigorous exclusion of water.

Using building blocks derived from cholic acids, we generally obtain very little diversity: there appears to be a strong thermodynamic driving force which produces very high yields of cyclic dimers and only small quantities of higher oligomers [15]. Individual dimers and trimers can be isolated, stereochemically characterized by nuclear Overhauser enhancement spectroscopy (NOESY) (and sometimes by crystallography), and can be induced to re-establish equilibrium by addition of catalytic acid. It remains unpredictable whether the configuration about the hydrazone linkage will turn out to be cis or trans (Fig. 12) [16]. When the monomers or dimers shown in Fig. 12 are mixed and induced to exchange by addition of acid, no mixed products are obtained: we simply see self-sorting into the two
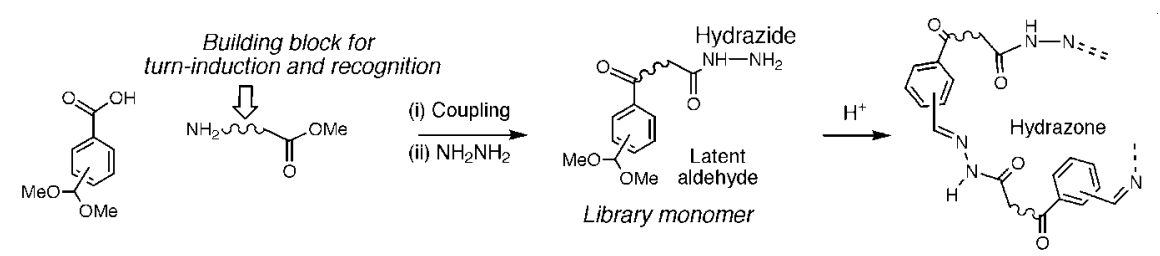

Fig. 11 General scheme for hydrazone libraries.
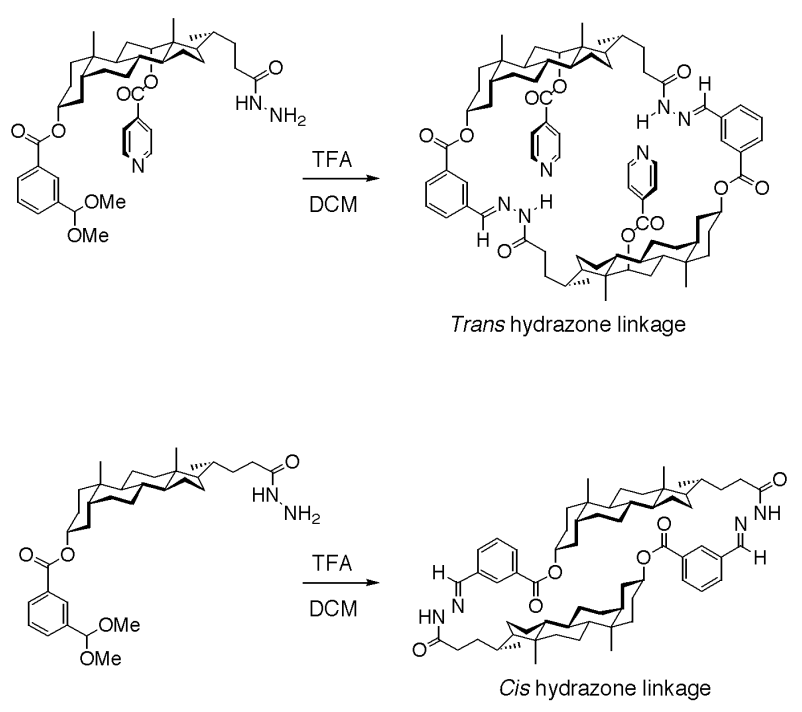

Fig. 12 Macrocyclic dimer formation from steroidal monomers. 
homodimers. This phenomenon, which occurs when there is a clear mismatch in the size and shape of reactive building blocks is a common observation in thermodynamically controlled chemistry $[13,14]$ : mixed intermediates are of course formed (Fig. 13) but are proofread and consumed. Such effects are of course not possible in conventional kinetically controlled synthesis. It has been possible to tempt cholate-derived into forming diverse combinatorial libraries with nucleoside and other flexible building blocks [16,17].

Using building blocks derived from amino acids, we can generate diverse libraries of oligomers, quench the exchange with base, and prove that addition of catalytic acid generates dynamic exchange (Fig. 14) [15]. Indeed, as exchange requires lower acid concentration than the initial acetal deprotection step we generally create mixed libraries from preformed libraries containing a single type of monomer.

Very recently, we have been able to demonstrate effective templating using quaternary ammonium and Li cations: these increase the proportion of cyclic trimer in certain peptide libraries from less than $10 \%$ to over $90 \%$, allowing the isolation and characterization of otherwise almost inaccessible material [18]. The precise mechanism is not yet clear, but trimer binding selectivity is confirmed in some cases by electrospray MS, and cation recognition by similar cyclic peptides has been reported

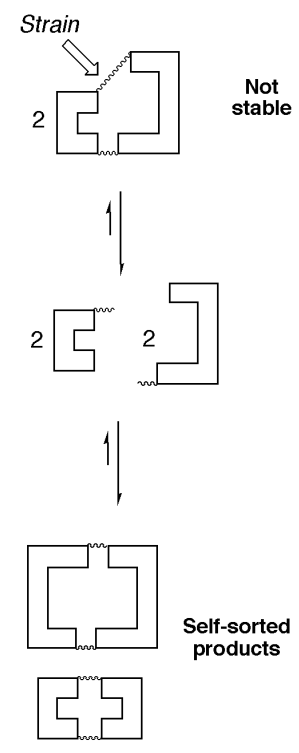

Fig. 13 Self-sorting in strained systems.

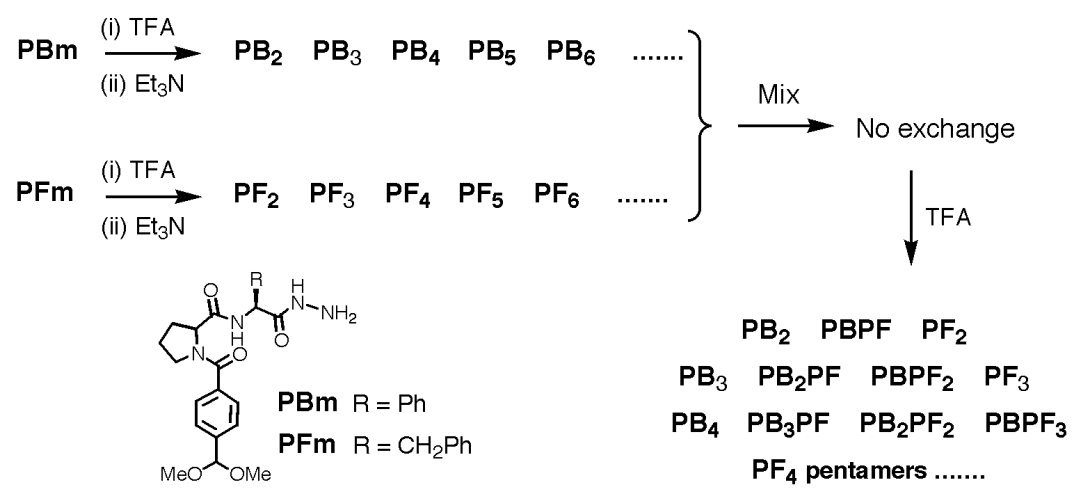

Fig. 14 Formation of cyclic oligomers of dipeptide hydrazones, and demonstration of the turning off and on of exchange; assay is by electrospray mass spectrometry [15].

(C) 2000 IUPAC, Pure and Applied Chemistry 72, 2265-2274 
elsewhere [19]. In any case, this is one of the first clear-cut examples in our work of successful, synthetic scale templating under thermodynamic conditions.

Disulfide exchange (Fig. 10e) is complementary to hydrazone exchange in that it is turned on under basic conditions and is turned off by acid. Preliminary results have confirmed the practicality of generating and exchanging disulfide libraries in water, and the search is now on for templating [20]. Another reaction that should prove effective in the future when the efficacy and lifetimes of catalysts have improved is alkene metathesis (Fig. 10c). We demonstrated that this could be used for the synthe-
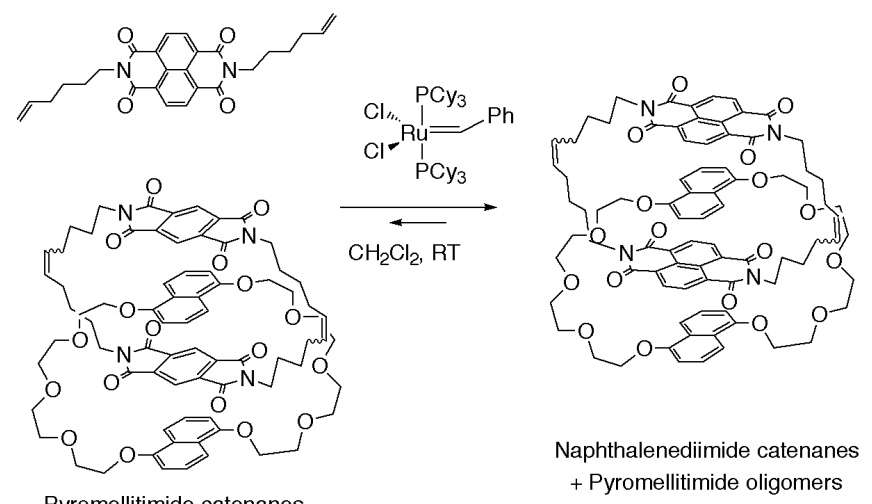

Fig. 15 Metathesis-induced exchange of components in catenanes [21].

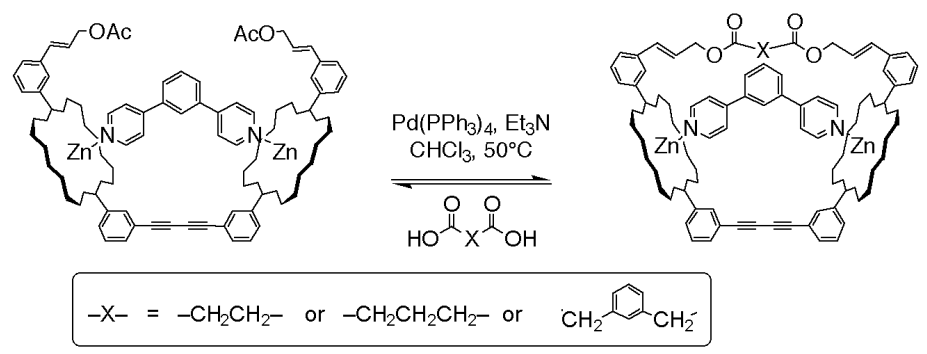

Fig. 16 Templated capping of a porphyrin dimer using Pd-catalyzed chemistry [23].

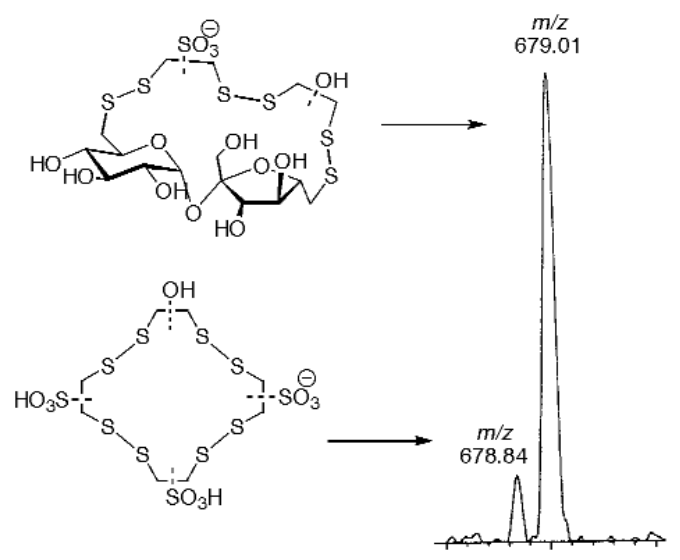

Fig. 17 Small part of the negative ion FT ICR mass spectrum of an aqueous disulfide library in water [20]. 
sis and then exchange of catenanes as summarized in Fig. 15 [21]. Leigh and co-workers independently carried out this "magic ring" experiment around the same time [22].

The final covalent reaction we are currently exploring brings us back to the question of how to generate new capped porphyrin dimers. One can imagine combining the design and selection approaches by taking a linear porphyrin dimer, bound template (which might be a TSA), and then inviting that complex to select its optimum cap from a mixture. The reaction we have chosen for exploring this possibility is Pd-catalyzed allyl transesterification (Fig. 10d) [23]. Indeed, it has been possible to isolate capped dimers as shown in Fig. 17, albeit in low yield; in the absence of template, or using kinetic chemistry, we have never been able to isolate any cyclic products from the attempted capping of linear dimers.

The stage is now set for expanding the range of building blocks using one or more of the general schemes in Fig. 10, attaching templates to beads and selecting good receptors. A key aspect of this phase of the project will be the use of appropriate methods for solving the formidable analytical challenges that face us. We have shown that gel-phase MAS NMR can elucidate the interactions between receptors and beads [24], and that Fourier transform ion cyclotron resonance (FTICR) mass spectrometry is a tremendously powerful tool for analysis of complex libraries [20,25]. Figure 16 illustrates this point by demonstrating the mass resolution, and thus the structural resolution, that is available: the two sets of compounds illustrated differ in mass by just $0.17 \mathrm{amu}$.

\section{ACKNOWLEDGMENTS}

I am grateful to my many talented co-workers named in the references for contributing so much to our understanding of this area, and to the EPSRC, EU, MSD, Glaxo-Wellcome, Astra-Zeneca, and Ethyl for supporting our research in this area.

\section{REFERENCES}

1. J. K. M. Sanders. Chem. Eur. J. 4, 1378-1383 (1998).

2. H. L. Anderson and J. K. M. Sanders. J. Chem. Soc., Perkin Trans. I 2223-2229 (1995).

3. J. K. M. Sanders. The Porphyrin Handbook, K. M. Kadish, K. M. Smith, R. Guilard (Eds.), Vol. 3, pp. 347-368, Academic Press (2000).

4. S. Anderson, H. L. Anderson, J. K. M. Sanders. J. Chem. Soc., Perkin Trans. I 2247-2254 (1995).

5. Z. Clyde-Watson, A. Vidal-Ferran, L. J. Twyman, C. J. Walter, D. W. J. McCallien, S. Fanni, N. Bampos, R. S. Wylie, J. K. M. Sanders. New J. Chem. 22, 493-502 (1998).

6. H. L. Anderson, S. Anderson, J. K. M. Sanders. J. Chem. Soc., Perkin Trans. I 2231-2245 (1995).

7. M. Marty, Z. Clyde-Watson, L. J. Twyman, M. Nakash, J. K. M. Sanders. Chem. Commun. 2265-2266 (1998).

8. M. Nakash, Z. Clyde-Watson, N. Feeder, J. E. Davies, S. J. Teat, J. K. M. Sanders. J. Am. Chem. Soc. 122, 5286-5293 (2000); M. Nakash, J. K. M. Sanders. J. Org. Chem. 65, 7266-7271 (2000).

9. M. Nakash, Z. Clyde-Watson, N. Feeder, S. J. Teat, J. K. M. Sanders. Chem. Eur. J. 6, 2112-2119 (2000).

10. P. A. Brady and J. K. M. Sanders. Chem. Soc. Rev. 26, 327-336. (1997).

11. (a) J. M. Lehn. Chem. Eur. J. 5, 2455-2463 (1999); (b) G. R. L. Cousins, S.-A. Poulsen, J. K. M. Sanders. Curr. Opin. Chem. Biol. 4, 270-279 (2000).

12. P. A. Brady and J. K. M. Sanders. J. Chem. Soc., Perkin Trans. I, 3237-3254 (1997); structure corrections, J. Chem. Soc., Perkin Trans.1, 2119 (1998).

13. S. J. Rowan, P. S. Lukeman, D. J. Reynolds, J. K. M. Sanders. New J. Chem. 22, 1015-1018 (1998).

14. S. J. Rowan, D. J. Reynolds, J. K. M. Sanders. J. Org. Chem. 64, 5804-5814 (1999).

15. G. R. L. Cousins, S.-A. Poulsen, J. K. M. Sanders. Chem. Commun. 1575-1576 (1999).

(C) 2000 IUPAC, Pure and Applied Chemistry 72, 2265-2274 
16. M. G. Simpson, S. P. Watson, J. K. M. Sanders. Organic Letters 2, 1435-1438 (2000) and unpublished results.

17. M. G. Simpson, J.-C. Meillon, J. K. M. Sanders. Unpublished results.

18. G. R. L. Cousins, R. L. E. Furlan, Y.-F. Ng, J. E. Redman, J. K. M. Sanders. Angew. Chemie Intl. Edn. 40, 423-428 (2000), and unpublished results.

19. S. Kubik, R. Goddard. J. Org. Chem. 64, 9475 (1999).

20. S. Otto, R. L. E. Furlan, J. K. M. Sanders. J. Am. Chem. Soc. 122, 12063-12064 (2000).

21. D. G. Hamilton, N. Feeder, S. J. Teat, J. K. M. Sanders. New J. Chem. 22, 1019-1021 (1998).

22. T. J. Kidd, D. A. Leigh, A. J. Wilson. J. Am. Chem. Soc. 121, 1599-1600. (1999).

23. G. Kaiser and J. K. M. Sanders. Chem. Commun. 1763-1764 (2000).

24. Y. R. de Miguel, N. Bampos, K. M. N. de Silva, S. A. Richards, J. K. M. Sanders. Chem. Commun. 2267-2268 (1998).

25. S.-A. Poulsen, P. J. Gates, G. R. L. Cousins, J. K. M. Sanders. Rapid Commun. Mass Spectrom. 14, 44-48 (2000). 